\title{
Real Estate Pricing under Two-Sided Asymmetric Information
}

\author{
Jeremy Sandford ${ }^{1}$, Paul Shea ${ }^{2}$ \\ ${ }^{1}$ University of Kentucky, Lexington, USA \\ ${ }^{2}$ Bates College, Lewiston, USA \\ Email: jeremy.sandford@uky.edu,pshea@bates.edu \\ Received June 5, 2013; revised July 5, 2013; accepted July 15, 2013
}

Copyright (C) 2013 Jeremy Sandford, Paul Shea. This is an open access article distributed under the Creative Commons Attribution License, which permits unrestricted use, distribution, and reproduction in any medium, provided the original work is properly cited.

\begin{abstract}
What happens when a buyer and a seller each have private information about the value of an item for trade, as is particularly common in real estate? We solve for the equilibrium price under both public information, where the seller shares his information with the buyer, and private information, where the seller is constrained to be unable to credibly share. Our main results are 1) even under public information, the equilibrium price differs from the expected value of the item, 2) under private information, prices follow a step function, with small changes in information generically having no effect on price, and 3) equilibrium price is more sensitive to informational changes under private information than public information. This under-studied game of 2-sided asymmetric information reasonably describes real estate transactions.
\end{abstract}

Keywords: Asymmetric Information; Game Theory; Information

\section{Introduction}

A buyer and a seller bargain over an asset of uncertain value, such as real estate. Both have private information relevant to the value of the asset. The seller makes a takeit-or-leave-it offer, which the buyer either accepts or rejects. We characterize the function mapping the seller's private information into his optimal offer, under both public information (the seller credibly reveals his information) and private information. We find that even under public information the seller's optimal offer is less sensitive to variations in private information than is the expected value of the asset. Under private information, the game's only equilibrium is a step function, under which the seller charges one of several discrete prices, depending on his information. Most surprisingly, the average sensitivity of price to the seller's information is greater under private information than public information, meaning that the seller charges a relatively higher price given favorable information if his information is private. There is some evidence that the seller's profit is higher under public information, meaning that he would be willing to pay to credibly reveal his private information, even given the risk that this information will be unfavorable.

Markets in which both sides have private information have not been studied extensively. Some existing work has studied the propensity to settle a lawsuit when both sides have private information about their likelihood of success (Friedman and Wittman (2007) [1], Daughety and Reinganum (1994) [2]), bargaining over labor disputes (Kennan and Wilson (1993) [3]), or the setting of point spreads in gambling markets (Sandford and Shea (2013 [4]), Ottaviani and Sorenson (2006) [5], and Steele and Zidek (1980) [6]). This paper extends the framework of Sandford and Shea (2013) [4], which finds the unexpected result that bookmakers do not optimally set gambling lines so that each side is equally likely to win when both the bookmaker and gambler have private information, to a real estate market, in which buyers and sellers negotiate over the price of an asset of uncertain value ${ }^{1}$. Our results on the relationship between optimal price and whether information is public or private, and on the elasticity between price and information are novel to the literature.

\section{Model}

Seller and Buyer negotiate over an asset, such as real

${ }^{1}$ Previous papers on real estate markets do not consider the implications of asymmetric information. See, for example, Yavas (1992) [7], and Yavas and Yang (1995) [8]. 
estate. The value of the asset to Buyer is $X \in[0,1]$. Seller's value of retaining the asset is $X /(1+A)$, so the efficient outcome is for Seller to sell to Buyer. Both agents receive information relevant to $X$. For Seller, this signal may represent the information that they have acquired from having owned the property, or from getting a professional appraisal. For Buyer, this signal may result from his own appraisal, advice from his real estate agent, or his own preferences over type of house.

Formally, suppose that Seller draws information $z_{1} \in$ $[-1,1]$ while Buyer draws information $z_{2} \in[-1,1]$.

Conditional on both pieces of information, the true distribution and density of $X$ are given by $G\left(x \mid z_{1}, z_{2}\right)$ and $g\left(x \mid z_{1}, z_{2}\right)$, respectively. We assume that $G$ and $g$ have the following functional forms:

$$
\begin{aligned}
& G\left(x \mid z_{1}, z_{2}\right)=x\left(1-\frac{1}{2}\left(z_{1}+z_{2}\right)\right)+\frac{x^{2}}{2}\left(z_{1}+z_{2}\right) \\
& g\left(x \mid z_{1}, z_{2}\right)=1-\frac{1}{2}\left(z_{1}+z_{2}\right)+x\left(z_{1}+z_{2}\right)
\end{aligned}
$$

For the remainder of the paper we consider the case where Seller makes a take-it-or-leave-it offer to Buyer consisting of price $p$, which Buyer will either accept or reject, based on his private information. However, the model's results are qualitatively similar if we instead assume that Buyer makes the offer.

Buyer will accept the offer only if his information is sufficiently favorable, if $z_{2}>\bar{z}$ for some $\bar{z}$ which depends on $\mathrm{p}$ and, if known to Seller, $z_{1}$. Formally, payoffs are then as follows:

$$
\begin{aligned}
& \pi_{B}^{R}=0 \\
& \pi_{B}^{A}=E\left[X \mid z_{1}, z_{2}\right]-p \\
& \pi_{S}^{R}=\frac{E\left[X \mid z_{1}, z_{2} \leq \bar{z}\right]}{1+A} \\
& \pi_{S}^{A}=p
\end{aligned}
$$

Buyer's strategy consists entirely of a choice of $\bar{z}$, conditioned on $p$ and, if available, $z_{1}$. Seller's strategy consists of a price $p$, conditioned on $z_{1}$. We first consider the case of public information, where $z_{1}$ is public information, known to both Buyer and Seller.

\subsection{Public Information}

Suppose Seller's information $z_{1}$ is known to Buyer, while $z_{2}$ is Buyer's private information. This may represent a case, for example, where Buyer's appraisals are able to successfully reveal all relevant information about the property, where Seller can commit to truthfully revealing his information, or it can emerge endogenously from a model in which Seller chooses whether or not to disclose and Buyer believes that any Seller who doesn't disclose $z_{1}$ has very bad information (low $z_{1}$ ), and so Seller is always better off by disclosing $z_{1}$, regardless of its value.

Suppose that both $z_{1}$ and $z_{2}$ are independently uniformly distributed over $[-1,1]$, that is $z_{1}, z_{2} \sim U[-1,1]$. We analyze the game backwards, first determining how Buyer sets $\bar{z}\left(p, z_{1}\right)$ and then how Seller sets $p\left(z_{1}\right)$. First, given $z_{1}$, Buyer is better off accepting an offer of $p$ if $\pi_{B}^{A} \geq \pi_{B}^{R}$. In the case of $z_{2}=\bar{z}\left(p, z_{1}\right)$, Buyer is indifferent between accepting and rejecting the offer, while he accepts (rejects) for $\mathrm{z}_{2}$ greater than (less than) $\bar{z}\left(p, z_{1}\right)$. Therefore, $\bar{z}$ is defined implicitly by (1).

$$
E\left[X \mid z_{1}, \bar{z}\left(p, z_{1}\right)\right]=p
$$

For very high (very low) prices $\mathrm{p}$, Buyer always rejects (always accepts). For intermediate prices, Lemma 1 establishes a functional form for $\bar{z}$ and shows that $\bar{z}$ is linearly increasing in $\mathrm{p}$ and linearly decreasing in $z_{1}$.

Lemma 1: Under public information, given information $z_{1}$ Buyer optimally accepts an offer of $p$ if and only if $z_{2} \geq \bar{z}\left(p, z_{1}\right)$, where:

$$
\bar{z}\left(p, z_{1}\right)= \begin{cases}1 & \text { if } p>\frac{7}{12}+\frac{1}{12} z_{1} \\ 12 p-z_{1}-6 & \text { if } p \in\left[\frac{5}{12}+\frac{1}{12} z_{1}, \frac{7}{12}+\frac{1}{12} z_{1}\right] \\ -1 & \text { if } p<\frac{5}{12}+\frac{1}{12} z_{1}\end{cases}
$$

Proof: Performing the integration in (1):

$$
\begin{aligned}
& E\left[X \mid z_{1}, \bar{z}\left(p, z_{1}\right)\right] \\
& =\int_{0}^{1} x\left(1-\frac{1}{2}\left(z_{1}+\bar{z}\right)+x\left(z_{1}+\bar{z}\right)\right) \mathrm{d} x=p \\
& \rightarrow \frac{x^{2}}{2}-\frac{x^{2}}{4} z_{1}-\frac{x^{2}}{4} \bar{z}+\frac{x^{3}}{3} z_{1}+\left.\frac{x^{3}}{3} \bar{z}\right|_{0} ^{1} \\
& \rightarrow \frac{1}{2}-\frac{1}{4} z_{1}-\frac{1}{4} \bar{z}+\frac{1}{3} z_{1}+\frac{1}{3} \bar{z}=p \\
& \rightarrow \bar{z}\left(p, z_{1}\right)=12 p-z_{1}-6
\end{aligned}
$$

Equation (2) and the fact that $z_{2}$ is bounded between -1 and 1 establish the lemma. $\square$

Seller takes Buyer's optimal strategy $\bar{z}\left(p, z_{1}\right)$ as given in setting $p$. In maximizing his payoff, Seller considers the gain in setting a higher price (higher profit in the event Buyer accepts) against the cost (lower probability of acceptance). First, it is immediate that for sufficiently low A, Seller will never sell-he will set a high $p$ so that Buyer rejects the offer. This is because for low A, Buyer accepts only if his private information suggests the value of the asset is worth more than $p$, in which case Seller would be better off keeping the land for himself. Second, it is clear that Seller's optimal price must be 
increasing in $z_{1}$; as Seller has more favorable public information, Buyer's willingness to pay and Seller's opportunity cost of selling both increase. Lemma 2 solves for Seller's optimal strategy $p\left(z_{1}\right)$ and formalizes the above claims.

Lemma 2: Under public information, Seller optimally sets a price of $p^{*}\left(z_{1}\right)$ where:

$$
p^{*}\left(z_{1}\right)=\frac{7+z_{1}}{24-\frac{12}{1+A}}
$$

$p^{*}$ is increasing in $z_{1}$ and decreasing in A. As A approaches zero, $\bar{z}\left(p^{*}\left(z_{1}\right), z_{1}\right)$ approaches 1 for all $z_{1}$.

Proof: Given $z_{1}$, Seller chooses $p$ to maximize expected profit,

$$
\begin{aligned}
& \operatorname{Pr}\left(z_{2}<\bar{z}\left(p, z_{1}\right)\right) \frac{E_{z_{2}}\left(X \mid z_{1}, z_{2}<\bar{z}\right)}{1+A} \\
& +\operatorname{Pr}\left(z_{2} \geq \bar{z}\left(p, z_{1}\right)\right) * p \\
& =\int_{-1}^{\bar{z}} \int_{0}^{1} \frac{1}{A} x g\left(x \mid z_{1}, z_{2}\right) \mathrm{d} x \frac{1}{2} \mathrm{~d} z_{2}+\int_{\bar{z}}^{1} \frac{1}{2} \mathrm{~d} z_{2} \\
& =\frac{1}{1+A} \int_{-1}^{\bar{z}} \frac{1}{4}+\frac{1}{24}\left(z_{1}+z_{2}\right) \mathrm{d} z_{2}+\frac{p(1-\bar{z})}{2} \\
& =\frac{1}{1+A}\left(\frac{1}{4} \bar{z}+\frac{1}{24} z_{1} \bar{z}+\frac{1}{48} \bar{z}^{2}+\frac{11}{48}+\frac{1}{24} z_{1}\right)+\frac{p(1-\bar{z})}{2} \\
& =\frac{1}{4(1+A)}\left(\bar{z}+\frac{1}{6} z_{1} \bar{z}+\frac{1}{12} \bar{z}^{2}+\frac{11}{12}+\frac{1}{6} z_{1}\right)+\frac{p(1-\bar{z})}{2}
\end{aligned}
$$

Taking the derivative of (4) and setting to zero then yields (3). That $p^{*}\left(z_{1}\right)$ is increasing in $z_{1}$ and decreasing in A follows from inspection of (3). That $x\left(p^{*}\left(z_{1}\right), z_{1}\right)=1$ for sufficiently low A follows from inserting $p^{*}$ from (3) into (2).

We analyze the public information equilibrium by comparing $p^{*}\left(z_{1}\right)$ to two bench marks. First, we show that $p^{*}\left(z_{1}\right)$ has no consistent relationship with $E\left(X \mid z_{1}\right)$. Second, in the next section, we compare the public information $p^{*}\left(z_{1}\right)$ with its counterpart when $z_{1}$ is Seller's private information, and cannot be credibly revealed.

First, a straightforward calculation shows that:

$$
E\left[X \mid z_{1}\right]=\frac{1}{2}+\frac{1}{12} z_{1}
$$

Consider the value of $p^{*}-E\left[X \mid z_{1}\right]$, calculated from (3) and (5):

$$
p^{*}-E\left[X \mid z_{1}\right]=\frac{1-5 A}{12(1+2 A)}-z_{1} \frac{A}{12(1+2 A)}
$$

Equation (6) is decreasing in $z_{1}$; Seller extracts a higher price relative to the expected value of the asset when his information is unfavorable. To put another way, Seller's optimal price is less elastic in his own informa- tion than is the expected value of the asset. Figure 1 demonstrates an example where $A=0.2$, implying that Seller obtains $25 \%$ less value from the property than Buyer, and that the average of $p^{*}-E\left[X \mid z_{1}\right]$ from (6) is 0 . That $p^{*}\left(z_{1}\right)$ is flatter than $E\left[X \mid z_{1}\right]$ reflects the greater price sensitivity of the latter.

\subsection{Private Information}

We now consider the case of private information where $z_{1}$ is known only to Seller, and $z_{2}$ only to Buyer. In this case, the Seller's private knowledge from owning the property cannot be fully extracted by Buyer, and by assumption, Seller cannot credibly reveal $z_{1}$ to buyer.

We again solve the game backwards. Upon observing a price set by Seller, Buyer forms beliefs over the distribution of $z_{1}$. Call this belief $f\left(z_{1}\right)$, and let $z_{1}^{e}(p)$ denote Buyer's expected value of $z_{1}$ based on a price of $p$. Again, Buyer will optimally accept an offer if and only if hissignal $z_{2}$ is above some threshold, $\bar{z}(p)$, defined implicitly by:

$$
E\left[X \mid f\left(z_{1}\right), \bar{z}\right]=p
$$

Lemma 3 establishes an analogue of Lemma 1 under private information, and describes the cutoff value of $z_{2}$, above which Buyer accepts and below which Buyer rejects. $\bar{z}$ is shown to depend positively on $p$ and negatively on $z_{1}^{e}$.

Lemma 3. Under private information, Buyer optimally accepts an offer of $p$ if and only if $z_{2} \geq \bar{z}(p)$, where:

$$
\bar{z}\left(p, z_{1}\right)= \begin{cases}1 & \text { if } p>\frac{7}{12}+\frac{1}{12} z_{1}^{e}(p) \\ 12 p-z_{1}^{e}(p)-6 & \text { if } p \in\left[\frac{5}{12}+\frac{1}{12} z_{1}^{e}(p), \frac{7}{12}+\frac{1}{12} z_{1}^{e}(p)\right] \\ -1 & \text { if } p<\frac{5}{12}+\frac{1}{12} z_{1}^{e}(p)\end{cases}
$$

Proof: Consider (7). Taking the expectation across both $X$ and $z_{1}$, we get:

$$
E\left[X \mid f\left(z_{1}\right), \bar{z}\right]=\int_{-1}^{1} f\left(z_{1}\right) \int_{0}^{1} x g(x) \mathrm{d} x \mathrm{~d} z_{1}
$$

Given the result in the proof of Lemma 1, (8) reduces to:

$$
\begin{aligned}
& E\left[X \mid f\left(z_{1}\right), \bar{z}\right] \\
= & \int_{-1}^{1} f\left(z_{1}\right)\left(\frac{1}{2}+\frac{1}{12} z_{1}+\frac{1}{12} \bar{z}\right) \mathrm{d} z_{1} \\
= & \frac{1}{2}+\frac{1}{12} z_{1}+\frac{1}{12} z_{1}^{e}
\end{aligned}
$$

The lemma follows from setting (9) equal to the price 


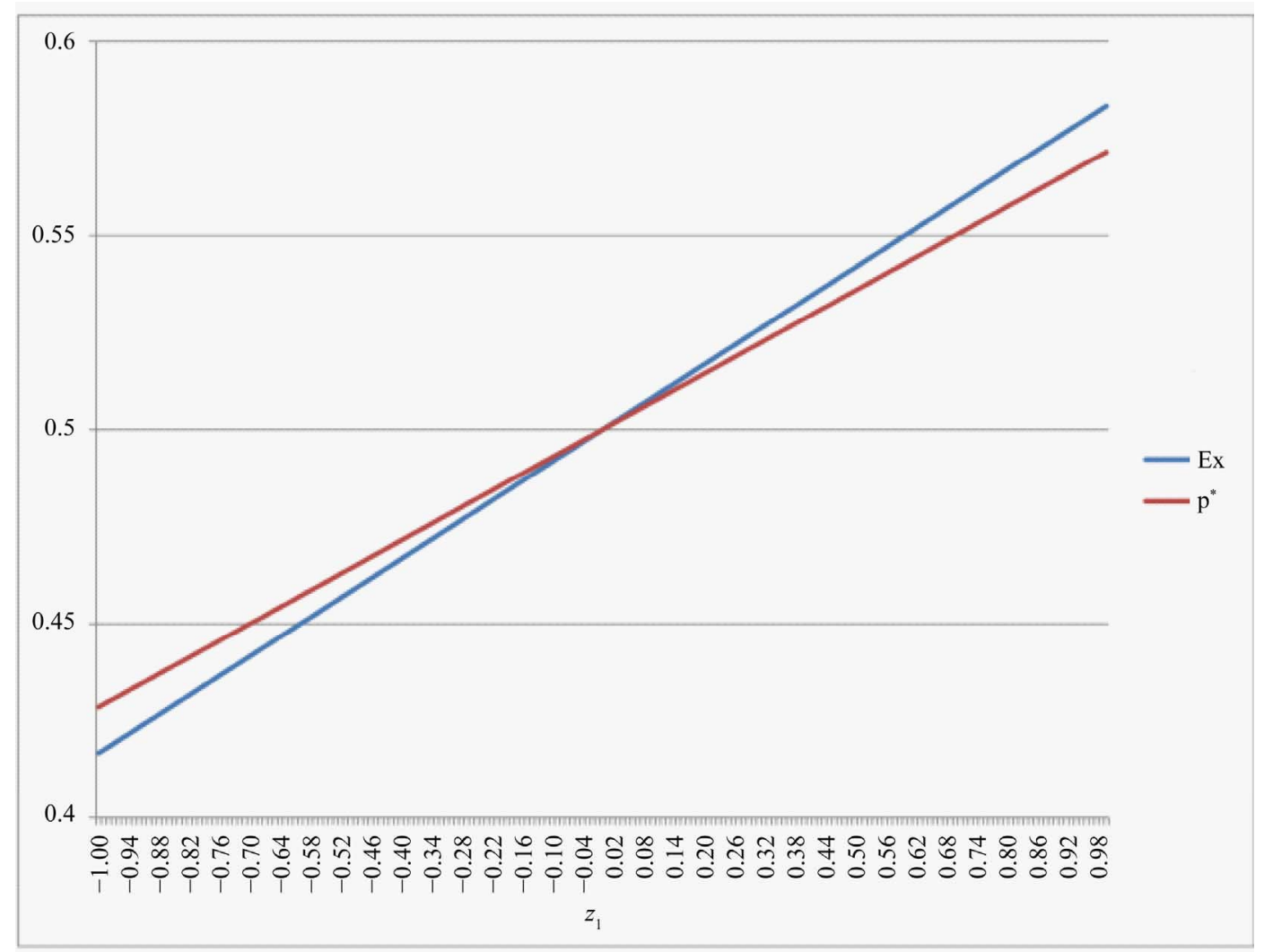

Figure 1. $E\left[X \mid z_{1}\right]$ and $p^{*}\left(z_{1}\right)$ for all possible $z_{1}$.

set by Seller, $p$. $\square$

Note from Lemma 3 that it is immaterial what the perceived probability distribution over different values of $z_{1}$ is; only the expectation matters. This follows from Seller's assumed risk neutrality.

Given Buyer's beliefs $z_{1}^{e}(p)$ and strategy $\bar{z}(p)$, Seller faces a trade off between a higher price and greater profit from a sale, and lower price and greater likelihood of sale. Formally, following (4) from the proof of Lemma 2 , for each $z_{1}$ Seller solves the following optimization problem:

$$
\begin{aligned}
\operatorname{Max}_{p} & \frac{1}{4(1+A)}\left(\bar{z}(p)\left(1+\frac{z_{1}}{6}\right)+\frac{1}{12} \bar{z}(p)^{2}+\frac{11}{12}+\frac{z_{1}}{6}\right) \\
& +p \frac{1-\bar{z}(p)}{2}
\end{aligned}
$$

Unsurprisingly, Seller's public information equilibrium strategy identified in Lemma 2 does not carry over to the case of private information; the temptation for Seller to shade his price to give Buyer a false impression of his private information is too great. Indeed, we show below that there is no equilibrium in which Seller's price is a linear function of his private information $z_{1}$. We cannot rule out exotic equilibrium functions, such as nonlinear functions $p\left(z_{1}\right)$. There are step equilibria of the following form: ${ }^{2}$

$$
p\left(z_{1}\right)= \begin{cases}p_{1} & \text { if } z_{1} \in\left[-1, \varsigma_{1}\right] \\ p_{2} & \text { if } z_{2} \in\left[\varsigma_{1}, \varsigma_{2}\right] \quad \ldots \\ p_{N} & \text { if } z_{2} \in\left[\varsigma_{N-1}, \varsigma_{N}\right]\end{cases}
$$

Lemma 4 proves that the equilibrium pricing function under privateinformation is not a linear function of $\mathrm{z}_{1}$.

Lemma 4: Under private information, no linear function of the form $p\left(z_{1}\right)=a+b z_{1}, b \neq 0$ solves Seller's optimization problem (10) over any subset of $z_{1} \in[-1,1]$.

Proof: Suppose that there did exist some linear function $p^{*}\left(z_{1}\right)=a+b z_{1}$ giving Seller's equilibrium price under private information, for some numbers $a$ and $b$. Noting that

$$
\frac{\partial}{\partial p}=12-\frac{1}{b}
$$

the first order condition for (10) is:

$$
\begin{aligned}
& \frac{1}{4(1+A)}\left(\left(12-\frac{1}{b}\right)\left(1+\frac{z_{1}}{6}\right)+\frac{\bar{z}}{6}\left(12-\frac{1}{b}\right)\right) \\
& +\frac{1-\bar{z}}{2}-p\left(12-\frac{1}{b}\right)=0
\end{aligned}
$$

\footnotetext{
${ }^{2}$ Step equilibria occur in other settings with asymmetric information, most notably Crawford and Sobel (1982) [9].
} 
Equation (12) in turn implies that:

$$
b=\frac{1}{24(1+A)-12-\frac{1}{b}}
$$

As (13) has no solution for $b \neq 0$, there does not exist a linear equilibrium pricing function for Seller, including the public information equilibrium.

Step equilibria always exist. In particular, if the number of steps is $N=1, x(p)=12 p-6$ and there is an equilibrium with trade under private information for any $p \in\left(\frac{7}{12(1+A)}, \frac{7}{12}\right)$. If $p>\frac{7}{12}$, even for $z_{2}=1$, Buyer will optimally reject any offer, while if $p<\frac{7}{12(1+A)}$, Seller will prefer to keep the item for $z_{1}$ $=1$. If, $A=0.5$ there is an equilibrium with $N=2$ where Seller plays $p_{1}=0.4715$ for all $z_{1} \in[-1,0]$ and $p_{2}=0.59$ for all $z_{2} \in(0,1]$. In general, there are a multiplicity of step equilibria for any number of steps $N$. However, for any value of $N$, the price of any one step $p_{i}$ uniquely determines the price at all other $N-1$ steps.

Table 1 gives example equilibria for $N \in\{1,3,5,7,9\}$. In each case, the equilibrium prices were chosen so that 1 ) the interval $[-1,1]$ is partitioned into segments of equal size and 2) the interval centered on 0 is priced at the same value. There does not appear to be a limit to the number of steps in an equilibrium, and Seller's profit does not appear to fluctuate wildly in the number of steps. All equilibria in the table are the unique equilibria with a price of 0.546 at the interval centered on 0 , but in eachcase a different price for this interval will produce a different equilibrium.

For any $N$, must be the case that at $\varsigma_{k}$ Seller is indifferent between $p_{k}$ and $p_{k+1}$ (if not, then he would surely also not be indifferent in some neighborhood

$\left(\varsigma_{1}-\varepsilon, \varsigma_{1}+\varepsilon\right)$. Seller's optimality then requires that $p_{2}$ be played for all $z_{1} \geq \varsigma_{1}$ and $p_{1}$ for all $\$ z_{1}<\varsigma_{1}$. Inspection of (4) gives us that this requires:

$$
x\left(p_{2}\right)>x\left(p_{1}\right) \leftrightarrow p_{2}-p_{1}>\frac{z_{2}^{e}-z_{1}^{e}}{12}
$$

Table 1. Different symmetric step equilibria, where $N$ is the number ofsymmetric steps.

\begin{tabular}{ccccccccccc}
\hline$N$ & $p_{1}$ & $p_{2}$ & $p_{3}$ & $p_{4}$ & $p_{5}$ & $p_{6}$ & $p_{7}$ & $p_{8}$ & $P_{9}$ & $E[\pi]$ \\
\hline 1 & 0.55 & & & & & & & & & 0.37 \\
3 & 0.47 & 0.55 & 0.61 & & & & & & & 0.37 \\
5 & 0.45 & 0.50 & 0.55 & 0.59 & 0.63 & & & & & 0.37 \\
7 & 0.44 & 0.48 & 0.52 & 0.55 & 0.58 & 0.60 & 0.63 & & & 0.37 \\
9 & 0.44 & 0.47 & 0.50 & 0.52 & 0.55 & 0.57 & 0.59 & 0.61 & 0.63 & 0.37 \\
\hline
\end{tabular}

Comparing Equations (14) and (15) leads to a surprising conclusion: under a step equilibrium, price is more elastic with respect to Seller's information when that information is private. Lemma 5 formalizes.

Lemma 5: Under any step equilibria of the form (11) on average the price charged by Seller increases more quickly in $z_{1}$ than the corresponding public information pricing function.

Proof: Referring back to the public information equilibrium price function, (3), we see that for any two prices $p_{2}>p_{1}$ charged in equilibrium,

$$
p_{2}-p_{1}>\frac{z_{2}-z_{1}}{24-\frac{12}{1+A}}
$$

From Equation (14) we see that the average rate of increase in a step equilibrium is $1 / 12$. The average rate of increase under public information is

$$
\frac{1}{24-\frac{12}{1+A}}<\frac{1}{12}
$$

Lemma 5 tells us that Seller's payoff from better information is higher if that information is private than if he always shares his information with Seller.

Figure 2 demonstrates the result of lemma 5 under $A=$ 0.5 , comparing public and private equilibrium pricing functions. Note the greater rate of increase under private information. In relative terms, Seller's profit increases by more upon a high draw of $z_{1}$ under private information. In this case, the expected profit under private information is 0.373 , while under public information it is 0.419 , suggesting that if Seller has the ability to credibly reveal his private information, he is better off doing so.

It is surprising that the case of private information, where Seller is able to manipulate Buyer's expectation, may result on a worse outcome for Seller. This result depends on our assumption that Seller cannot credibly reveal $z_{1}$.

Because the agents are bargaining over a surplus, they

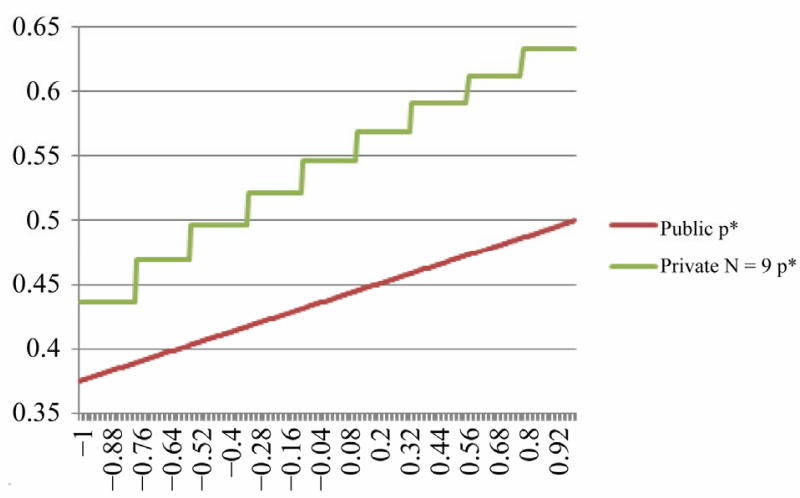

Figure 2. $E\left[X \mid z_{1}\right]$ and $p^{*}\left(z_{1}\right)$ for all possible $z_{1}$. 
have a mutual interest in reaching an agreement. By being unable to observe $z_{1}$, however, Buyer has worse information and it is more likely that a deal does not occur which reduces the average welfare of both agents.

\section{REFERENCES}

[1] D. Friedman and D. Wittman, "Litigation with Symmetric Bargaining and Two-Sided Incomplete Information," Journal of Law, Economics, and Organization, Vol. 23, No. 1, 2007, pp. 98-126. doi:10.1093/jleo/ewm004

[2] A. Daughety and J. Reinganum, "Settlement Negotiations with Two-Sided Asymmetric Information: Model Duality, Information Distribution, and Efficiency," International Review of Law and Economics, Vol. 14, No. 3, 1994, pp. 283-298. doi:10.1016/0144-8188(94)90044-2

[3] J. Kennan and R. Wilson, "Bargaining with Private Information," Journal of Economic Literature, Vol. 31, No. 1, 1993, pp. 45-104.

[4] J. Sandford and P. Shea, "Optimal Setting of Point Spreads," Economica, Vol. 80, No. 317, 2013, pp. 149-
170. doi:10.1111/j.1468-0335.2012.00939.x

[5] M. Ottaviani and P. Sorenson, "The Strategy of Professional Forecasting," Journal of Financial Economics, Vol. 81, No. 2, 2006, pp. 441-466. doi:10.1016/j.jfineco.2005.08.002

[6] J. M. Steele and J. Zidek, "Optimal Strategies for Second Guessers," Journal of the American Statistical Association, Vol. 75, No. 371, 1980, pp. 596-601. doi:10.1080/01621459.1980.10477519

[7] A. Yavas, "A Simple Search and Bargaining Model of Real Estate Markets," Real Estate Economics, Vol. 20, No. 4, 1992, pp. 533-548. doi:10.1111/1540-6229.00595

[8] A. Yavas and S. Yang, "The Strategic Role of Listing Price in Marketing Real Estate: Theory and Evidence," Real Estate Economics, Vol. 23, No. 3, 1995, pp. 374-368. doi:10.1111/1540-6229.00668

[9] V. Crawford and J. Sobel, "Strategic Information Transaction," Econometrica, Vol. 50, No. 6, 1982, pp. 14311451. doi: $10.2307 / 1913390$ 